Discussiones Mathematicae

\title{
A CHARACTERIZATION OF 2-TREE PROBE INTERVAL GRAPHS
}

\author{
DAVID E. BROWN \\ Department of Mathematics and Statistics \\ Utah State University \\ Logan, UT 84322, USA \\ e-mail: david.e.brown@usu.edu \\ Breeann M. Flesch \\ Mathematics Department \\ Western Oregon University \\ Monmouth, OR 97361, USA \\ e-mail: fleschb@wou.edu \\ AND \\ J. RiChARD LUNDGREN \\ Department of Mathematical Sciences \\ University of Colorado Denver \\ Denver, CO 80217, USA \\ e-mail: richard.lundgren@ucdenver.edu
}

\begin{abstract}
A graph is a probe interval graph if its vertices correspond to some set of intervals of the real line and can be partitioned into sets $P$ and $N$ so that vertices are adjacent if and only if their corresponding intervals intersect and at least one belongs to $P$. We characterize the 2 -trees which are probe interval graphs and extend a list of forbidden induced subgraphs for such graphs created by Pržulj and Corneil in [2-tree probe interval graphs have a large obstruction set, Discrete Appl. Math. 150 (2005) 216-231].
\end{abstract}

Keywords: interval graph, probe interval graph, 2-tree.

2010 Mathematics Subject Classification: 05C62, 05C75. 


\section{REFERENCES}

[1] L.W. Beineke, Characterizations of derived graphs, J. Combin. Theory 9 (1970) 129135. doi:10.1016/S0021-9800(70)80019-9

[2] L.W. Beineke and R.E. Pippert, Properties and characterizations of $k$-trees, Mathematika 18 (1971) 141-151. doi:10.1112/S0025579300008500

[3] D.E. Brown, Variations on Interval Graphs, PhD Thesis, (Univ. of Colorado, Denver, USA, 2004).

[4] D.E. Brown, A.H. Busch and G. Isaak, Linear time recognition algorithms and structure theorems for bipartite tolerance graphs and bipartite probe interval graphs, Discrete Math. Theor. Comput. Sci. 12(5) (2010) 63-82.

[5] D.E. Brown, S.C. Flink and J.R. Lundgren, Interval k-graphs, in: Proceedings of the Thirty-third Southeastern International Conference on Combinatorics, Graph Theory and Computing (Boca Raton, FL, 2002) 156 (2002) 5-16.

[6] D.E. Brown and L.J. Langley, Forbidden subgraph characterizations of bipartite unit probe interval graphs, Australas. J. Combin. 52 (2012) 19-31.

[7] D.E. Brown and J.R. Lundgren, Bipartite probe interval graphs, circular arc graphs, and interval point bigraphs, Australas. J. Combin. 35 (2006) 221-236.

[8] D.E. Brown, J.R. Lundgren and L. Sheng, A characterization of cycle-free unit probe interval graphs, Discrete Appl. Math. 157 (2009) 762-767. doi:10.1016/j.dam.2008.07.004

[9] P.C. Fishburn, Interval Orders and Interval Graphs (Wiley \& Sons, 1985).

[10] B. Flesch and J.R. Lundgren, A characterization of $k$-trees that are interval p-graphs, Australas. J. Combin. 49 (2011) 227-237.

[11] D.R. Fulkerson and O.A. Gross, Incidence matrices and interval graphs, Pacific J. Math. 15 (1965) 835-855.

doi:10.2140/pjm.1965.15.835

[12] M.C. Golumbic, Algorithmic Graph Theory and Perfect Graphs (Academic Press, New York, 1980).

[13] M.C. Golumbic and M. Lipshteyn, On the hierarchy of interval, probe and tolerance graphs, in: Proceedings of the Thirty-second Southeastern International Conference on Combinatorics, Graph Theory and Computing (Baton Rouge, LA, 2001) 153 (2001) 97-106.

[14] M.C. Golumbic and A.N. Trenk, Tolerance Graphs, Cambridge Studies in Advanced Mathematics, Vol. 18 (Cambridge University Press, Cambridge, 2004).

[15] C.G. Lekkerkerker and J.Ch. Boland, Representation of a finite graph by a set of intervals on the real line, Fund. Math. 51 (1962/1963) 45-64. 
[16] R. McConnell and Y. Nussbaum, Linear-time recognition of probe interval graphs, in: Proceedings of the 17th Annual European Symposium, Algorithms-ESA 2009, Lecture Notes in Comput. Sci. 5757 (2009) 349-360.

doi:10.1007/978-3-642-04128-0_32

[17] F.R. McMorris, C. Wang and P. Zhang, On probe interval graphs, Discrete Appl. Math. 88 (1998) 315-324. doi:10.1016/S0166-218X(98)00077-8

[18] A. Proskurowski, Separating subgraphs in k-trees: cables and caterpillars, Discrete Math. 49 (1984) 275-285. doi:10.1016/0012-365X(84)90164-X

[19] N. Pržulj and D.G. Corneil, 2-tree probe interval graphs have a large obstruction set, Discrete Appl. Math. 150 (2005) 216-231. doi:10.1016/j.dam.2004.06.015

[20] F.S. Roberts, Discrete Mathematical Models (Prentice-Hall, Upper Saddle River, NJ, 1976).

[21] L. Sheng, Cycle free probe interval graphs, in: Proceedings of the Thirtieth Southeastern International Conference on Combinatorics, Graph Theory, and Computing (Boca Raton, FL, 1999) 140 (1999) 33-42.

[22] P. Zhang, Methods of mapping DNA fragments, United States Patent, 1997 http://www.cc.columbia.edu/cu/cie/techlists/patents/5667970.htm

[23] P. Zhang, E.A. Schon, E. Cayanis, J. Weiss, S. Kistler and P.E. Bourne, An algorithm based on graph theory for the assembly of contigs in physical mapping of DNA, Comput. Appl. Biosci. 10(3) (1994) 309-317.

Received 3 December 2012

Revised 20 May 2013

Accepted 20 May 2013 